\title{
Observer Based Impedance Control of a Pneumatic System with Long Transmission Lines
}

\author{
Melih Turkseven ${ }^{1}$ and Jun Ueda ${ }^{2}$
}

\begin{abstract}
Pneumatic actuation has gained popularity in robotics, for its low maintenance, high efficiency and compactness. A recent direction is to build pneumatically driven non-magnetic platforms for robotic rehabilitation monitored by magnetic resonance imaging (MRI). Control of such systems is challenging as they involve long transmission lines to satisfy MR-compatibility requirements and direct pressure measurement at the actuator is not available. This paper presents a control algorithm that provides an accurate impedance control for pneumatic systems with long transmission lines. The algorithm involves a pressure observer based on force and displacement feedback to compensate for the lack of pressure measurement. The observed pressures are utilized in a robust controller for force tracking. The described method is tested on a system with 7 meters long transmission lines. Impedance control using the presented observer achieved stable force tracking up to $1.5 \mathrm{~Hz}$, lowering mean squared error by up to $\% 25$.
\end{abstract}

\section{INTRODUCTION}

The demand for rehabilitation providers increase with the aging population that has a higher risk of disability due to neurological disorders. As an example, the survivors of stroke, sudden loss of certain brain functions due to local damages in the brain tissue, regain the lost brain functions through long periods of repetitive exercises [1]. Robotic rehabilitation has become increasingly popular in facilitating functional recovery after this type of injury; since, the robots can reproduce physical exercises with great precision and extract quantitative feedback from the patients. An extensive reading by Krebs et al. summarizes the impact made by the robotic technology in this field [2]. Robots can be used to standardize physical therapies, increasing the success rate of a treatment that would, otherwise, depend on the manual skills of therapists.

A recent trend in robotic rehabilitation is to execute rehabilitation procedures in the magnetic resonance imaging (MRI) rooms, in order to utilize the high-resolution brain monitoring capability of MRI machines. The use of MR technology during the application of the treatment is considered to be a powerful method for both understanding the mechanisms of brain recovery and analyzing the efficacy of existing rehab procedures [3]. Several MRI-compatible, robotic platforms have been used in clinical studies and were shown to be beneficial [4].

\footnotetext{
*This work was supported by the Center of Compact and Efficient Fluid Power (CCEFP).

${ }^{1}$ Melih Turkseven is with the Department of Mechanical Engineering, Georgia Institute of Technology, 785 Ferst Dr. 30318, USA mturkseven3@gatech.edu

${ }^{1}$ Jun Ueda is with Faculty of Mechanical Engineering, Georgia Institute of Technology, 785 Ferst Dr. 30318, USA jun.ueda@me.gatech.edu
}

The major drawback of MRI is the limitations due to its strong magnetic field that prevents conventional electrical actuation systems to perform in the MRI room. Only materials within a certain range of the susceptibility spectrum are allowed [5]. Fluid driven actuation systems have been considered to provide ultimate MR compatibility compared to other non-ferrous alternatives [6]. These sort of systems are generally powered through transmission lines between the MR-compatible actuator that lies in the MRI room and the ferro-magnetic drivers that stay outside the room [4]. Pneumatic actuation, in particular, has a number of convenient practical properties for haptic operations in healthcare units. A detailed comparison between the hydraulic and pneumatic actuation systems was presented by Yu et al. [7]. Compared to hydraulic systems, pneumatic cylinders are compact, low-cost and easy to maintain systems. They are also back-drivable, offering an inherent compliance which is safe for haptic interactions. In addition, an auxiliary air source is generally available in medical facilities. However, such systems have a complicated dynamics due to the compressibility of air and the presence of transmission lines between the air source and the actuators. Direct pressure reading at the actuators in MRI is not available due to the MRI safety requirements. Therefore, the control of such teleoperated pneumatic systems demand extra attention.

Therapeutic robots are, in essence, haptic interfaces that would maintain a desired contact impedance as the therapy is applied. A fast dynamic response is required to reproduce the desired transient forces of contact events. Therefore, accurate force and position feedback obtained at the contact point are very crucial for a satisfactory performance. Commercially available optical encoders have been in use for displacement feedback in MRI. In the recent years, several MRI compatible optical force sensors have been developed [8]. However, pneumatic actuation has been exploited in a limited number of rehabilitation-related studies so far [9]. When coupled with MRI-safe sensory units; pneumatically driven tele-operated platforms can be utilized to provide a controlled impedance on haptic interactions.

\section{PROBLEM STATEMENT}

Pneumatic actuation is suitable for medical applications for their low maintenance, compactness and efficiency. However, the control of pneumatic actuators becomes difficult when the system involves long transmission lines between the air source and the actuators, such as in the case of MRI-compatible robots [7]. To improve the performance of pneumatically actuated systems, pressure information is 


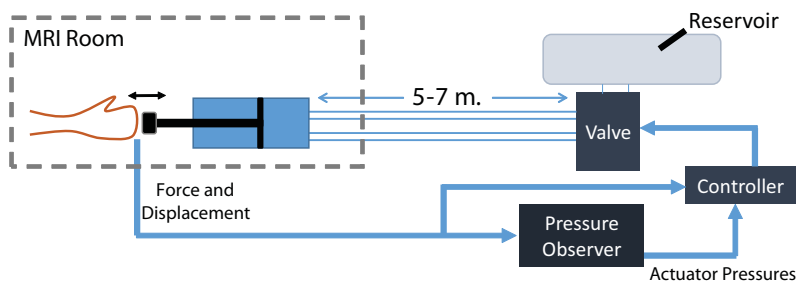

Fig. 1: The schematics of the developed system for the intended application

commonly used and combined with a controller. When direct pressure measurement is not available or not desirable, such as in this application, pressures can be estimated by a model of the system [10].

Obtaining accurate information on the pressure states of a tele-operated system is difficult for structural reasons. Direct pressure reading in MRI via pressure sensors is not available, due to the strict material limitations for MR compatibility. Open-loop estimation also yields limited accuracy. Pressure attenuation and time-delay introduced by long transmission lines make it difficult to apply conventional models for pressure estimation and control [11]. In addition, such models unfortunately cannot fully characterize additional hardwarespecific complex dynamics such as frictional losses or leakage in the equipment [12] For MRI compatibility, glass cylinders with carbon pistons are being used. In addition to low magnetic susceptibility, glass cylinders can achieve low friction, yet the gas leakage through their ports are not negligible.

In this study, an asymptotically stable pressure observation algorithm that utilizes force and displacement feedback is used to compensate for the lack of direct pressure reading, as shown in Fig. 1. The proof of stability in the observer dynamics has been provided by the authors in an earlier study [13]. The observed pressure states are provided to a sliding-mode based non-linear controller. The suitability of this control scheme is tested on a 1-DOF pneumatic system with sufficiently long transmission lines for MRI related applications. This manuscript is organized to describe the observer and the controller, followed by impedance control tests on the experimental setup.

\section{PNEUMATIC SYSTEM MODEL}

\section{A. Standard System Model}

This section outlines an existing model of each component. The combination of these individual models is referred as the standard model in the rest of the paper.

1) Valve: The mass flow through a pneumatic valve is widely characterized as an isentropic flow through a frictionless orifice. The following relation between the area of the valve orifice and mass flow rate is given assuming steadystate boundary conditions [14]:

$$
\dot{m}_{v_{i}}=\operatorname{sign}\left(A_{v_{i}}\right) C_{A_{v_{i}}} \psi\left(P_{u}, P_{d}\right)
$$

where $A_{v_{i}}$ is the cross-section area of $i^{t h}$ port of the valve at a given input command, and $\psi$ is a piecewise function of the upstream $\left(P_{u}\right)$ and downstream $\left(P_{d}\right)$ pressures across the valve. $C_{A_{v_{i}}}$ is the sonic conductance of the valve that is characterized by a low order polynomial of the orifice area, $A_{v_{i}} . \psi$ is defined as follows:

$$
\psi\left(P_{u}, P_{d}\right)= \begin{cases}P_{u} \rho_{0} \sqrt{\frac{T_{0}}{T}} & \text { if } \frac{P_{d}}{P_{u}} \geq b \\ P_{u} \rho_{0} \sqrt{\frac{T_{0}}{T}} \sqrt{1-\left(\frac{P_{d}}{P_{u}-b}\right)^{2}} & \text { if otherwise }\end{cases}
$$

where $T$ is the stagnation temperature of the gas, $\rho_{0}$ and $T_{0}$ are density and temperature of air at reference conditions respectively [15], $b$ is the critical ratio of the pressures that determines whether the flow is choked or unchoked, and $R$ is the ideal gas constant. As shown in (1), the direction of the flow is represented with the sign of the area where a positive value means the chamber of the actuator is being charged and negative means the chamber is discharged given as:

$$
\psi\left(P_{u}, P_{d}\right)= \begin{cases}\psi\left(P_{\text {reservoir }}, P_{\text {actuator }}\right), & \text { if } A_{v} \geq 0 \\ \psi\left(P_{\text {actuator }}, P_{\text {atm }}\right), & \text { if otherwise }\end{cases}
$$

where $P_{\text {reservoir }}$ is the reservoir pressure, $P_{\text {actuator }}$ is the chamber pressure and $P_{a t m}$ is the ambient pressure.

2) Chamber Dynamics: The dynamic relationship between the displacement of the actuator piston and valve input can be characterized by the mass flow rate. Using the ideal gas law and continuity equation, the following dynamic expression of the chamber pressure is obtained:

$$
r \dot{m} R T=r P \dot{V}+\dot{P} V
$$

where $r$ varies from 1 to 1.4 depending on the level of heat transfer when the chamber is being charged or discharged. $r$ becomes 1 for the isothermal process and 1.4 for the adiabatic [16]. The volume of the chamber being charged/discharged is represented by $V$ :

$$
V=V_{\text {actuator }}+V_{\text {line }}
$$

with $V_{\text {actuator }}$ as the chamber volume and $V_{\text {line }}$ as the fixed volume of the transmission line that is connected to that chamber.

By combining the volume of the transmission line and actuator chamber, this model assumes a homogeneous pressure distribution along the system beyond the valve. This is one of the main reasons a closed-loop routine for pressure observation is necessary for high accuracy.

3) Piston Dynamics: The piston of a pneumatic actuator is displaced by the pressure difference between the chamber pressures, shown in Fig 2. The magnitude of the force produced by an actuator, referred as actuator force, is given by:

$$
F_{p}=P_{1} A_{1}-P_{2} A_{2}-P_{a t m} A_{p}
$$

where $P_{a t m}$ represents the atmospheric pressure and $A_{p}$ is the area of the piston rod.

The following equation represents the dynamics of a piston by relating the actuator force to the displacement of the piston:

$$
F_{p}=F_{e}+M \ddot{z}+B \dot{z}+F_{c} \operatorname{sign}(\dot{z})
$$




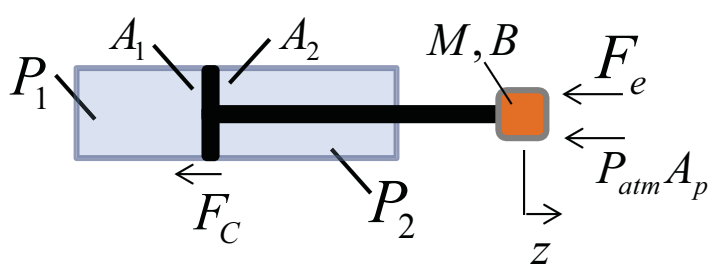

Fig. 2: Free-body diagram of the actuator piston

The piston impedance is represented by the inertia and damping parameters $M$ and $B$, illustrated in Fig. 2. The displacement of the piston from the mid-stroke $\frac{L}{2}$ is represented by $z$. The external force, $F_{e}$, can be measured by using a force sensor attached to the piston rod. In our study, possible coulomb friction across the piston, $F_{c}$, is neglected for the actuator being utilized has a graphite piston that produces negligible friction.

\section{PRESSURE OBSERVER}

The standard pneumatic system model outlined in the previous section oversees the additional dynamics on a teleoperation setup introduced by transmission lines. In the absence of pressure sensors, the accuracy of pressure observation via standard model can be improved by measuring force and displacement at the piston.

\section{A. Proposed Observer Dynamics}

In the proposed observation method, state variables are chosen as the stored energy in the system [10]:

$$
x=\left[\begin{array}{l}
x_{1} \\
x_{2}
\end{array}\right]=\left[\begin{array}{l}
P_{1} V_{1} \\
P_{2} V_{2}
\end{array}\right]
$$

Since the volume of each chamber can be easily calculated from the position sensor reading, individual chamber pressures can be obtained by:

$$
P_{1}=\frac{x_{1}}{V_{\text {line }}+\left(\frac{L}{2}+z\right) A_{1}} \quad P_{2}=\frac{x_{2}}{V_{\text {line }}+\left(\frac{L}{2}-z\right) A_{2}}
$$

where $P_{1,2}$ is the pressure in an actuator chamber, $A_{1,2}$ is the piston area and $L$ is the stroke length of the cylinder stroke shown in Fig. 2.

Considering the chambers as isothermal, i.e. $r=1$ in (4), the derivative of the states can be modeled by flow rates [10]. Measured force and piston displacement are utilized to correct errors in the estimated state variables, $\widehat{x_{1,2}}$, and help them converge:

$$
\widehat{\dot{x}}=\left[\begin{array}{c}
\widehat{\dot{x}_{1}} \\
\widehat{\dot{x}_{2}}
\end{array}\right]=\left[\begin{array}{c}
\widehat{\dot{m}_{1}} R T \\
\widehat{\dot{m}_{2}} R T
\end{array}\right]+k\left[\begin{array}{c}
f_{1}\left(\widehat{F_{p}}-F_{p}\right) \\
f_{2}\left(\widehat{F_{p}}-F_{p}\right)
\end{array}\right]
$$

where $\widehat{m_{1,2}}$ represent estimated mass flow rates. $k$ is a positive constant, and $\widehat{F_{p}}$ is the observed actuator force calculated from estimated pressures $\widehat{P_{1}}$ and $\widehat{P_{2}}$ by (6). The true actuator force, $F_{p}$, is realized by substituting force and position measurements into (7). The velocity and acceleration of the piston can be obtained by subsequent numerical derivations of the position measurement. The functions $f_{1}$ and $f_{2}$ are given as:

$$
\begin{aligned}
f_{1} & =-\frac{1}{V_{1} A_{2}}\left\{\hat{P}_{1} A_{1}-\hat{P}_{2} A_{2}-P_{a t m} A_{p}\right. \\
& \left.-\left(F_{e}+M \ddot{z}+B \dot{z}\right)\right\} \\
f_{2} & =+\frac{1}{V_{2} A_{1}}\left\{\hat{P}_{1} A_{1}-\hat{P}_{2} A_{2}-P_{a t m} A_{p}\right. \\
& \left.-\left(F_{e}+M \ddot{z}+B \dot{z}\right)\right\}
\end{aligned}
$$

The proposed observer given in (10) is comprised of estimated mass flow rates and correction terms based on force and displacement measurements. The flow rates are calculated by using estimated pressures on (1)-(3). The correction terms minimize the difference between estimated actuator force, $\hat{F}_{p}$, and true actuator force, $F_{p}$. To achieve asymptotic stability in the observer, the following control scheme is introduced:

$$
A_{v_{1}} A_{v_{2}}<0
$$

This condition is common for generic valves to enable flow control of two chambers with single control input. Fourway valves inherently satisfy this condition. The proposed observer can still preserve its stability regardless of the condition of the mass flow to the system. In addition, the proposed observer guarantees a limited reduction in observation accuracy in the presence of measurement errors. The proof of the asymptotically stable pressure observation and the analysis of robustness has been provided by the authors in an earlier study [13].

\section{CONTROLLER}

The aim of the control algorithm is to make the contact force $F_{e}$, exerted by the environment, reflect a desired impedance behavior. A generic approach to implement a desired impedance characteristic is defining a desired output force with regard to the motion of the end effector [17], [18]:

$$
F_{d}=m_{d}\left(\ddot{z}{ }_{d}-\ddot{z}\right)+b_{d}\left(\dot{z}_{d}-\dot{z}\right)+k_{d}\left(z_{d}-z\right)
$$

where $m_{d}, b_{d}, k_{d}$ are specified inertia, damping and stiffness coefficients that define the desired impedance dynamics with respect to an equilibrium position $z_{d} . F_{d}$ represents the contact force at the desired state. For this study, a critically damped impedance characteristic is selected as the reference.

The efficacy of the presented observation scheme has been demonstrated by the authors, in an earlier study that involves a simple PI controller for force control [13]. As noted, the actuator pressures are generally used as states in the modelbased controller designs. This study aims to investigate the contribution of the improvement in pressure observation to the control accuracy. For this reason, a standard slidingmode based controller is utilized to satisfy the desired contact force. The sliding surface is selected as: $s=e+\lambda \int e d t$, where $e=F_{d}-F_{e}$.

The design of the control law that provides the desired output motion, represented by $s=0$, is well-defined and widely applied on the control of pneumatic actuators [19]. 


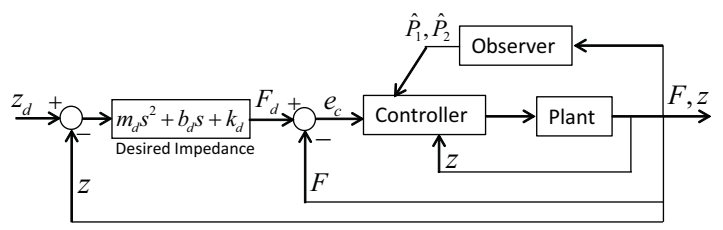

Fig. 3: The block diagram of the developed impedance controller

Therefore, the derivation of the valve input will be briefly described here and proofs for the controller stability are omitted.

The error dynamics is stabilized by choosing a Lyapunov candidate, $V=\frac{1}{2} s^{2}$ and setting its time derivative as a negative definite function, or $s \dot{s}<0$. This relation is satisfied by calculating an equivalent valve input that correspond to $\dot{s}=0$ according to the valve model and adding a robustness term. Based on the definition of the sliding surface, $s$, its derivative can be represented as:

$$
\dot{s}=\dot{F}_{d}-\dot{F}+\lambda\left(F_{d}-F\right)
$$

The value of the sonic conductance, $C_{A_{v}}$, that will set the derivative of $s$ to the targeted value can be calculated substituting (1)-(7):

$$
C_{A_{v_{e q}}}=\frac{\dot{F}_{d}+M \dddot{z}+B \ddot{z}+\frac{\hat{P}_{1} \dot{V}_{1} A_{1}}{V_{1}}-\frac{\hat{P}_{2} \dot{V}_{2} A_{2}}{V_{2}}}{\operatorname{sign}\left(A_{v_{e q}}\right) R T\left(\psi\left(P_{u}, \hat{P}_{1}\right) \frac{A_{1}}{V_{1}}+\psi\left(P_{u}, \hat{P}_{2}\right) \frac{A_{2}}{V_{2}}\right)}
$$

Then the equivalent valve input value, $A_{e q}$, that correspond to the calculated sonic conductance is solved utilizing the calibration of the valve. Note that the observed pressure states are substituted for the derivation of the equivalent input. The resultant input value, including the robustness term becomes:

$$
A_{v}=A_{v_{e q}}-\eta \operatorname{sat}\left(\frac{s}{\phi}\right)
$$

where $\eta$ is strictly positive to ensure error convergence to zero. To avoid chatter around the equilibrium, the robustness term involves a saturation function of $s$ over a boundary layer value of $\phi[19]$.

\section{EXPERIMENTAL SETUP}

A double acting cylinder (Airpel E9.0DU) was connected to a four-way spool valve (Enfield Tech. LH05) via 7.25 $\mathrm{m}$. long transmission lines of $4 \mathrm{~mm}$ inside diameter. The actuator pressures were measured by analog pressure sensors (SSI Technologies P51 series) at the cylinder ports. The pressure measured at the inlet of each actuator chamber was regarded as true pressure in the corresponding chamber. The external force applied to the actuator was measured by a load cell (FUTEK LSB200) attached at the tip of the piston rod. The piston was placed in front of a spring system that provided an unknown external force upon interaction, as shown in Fig. 4. The piston position was measured by using a Honeywell linear potentiometer (model F38000106). The velocity and acceleration of the piston are obtained by

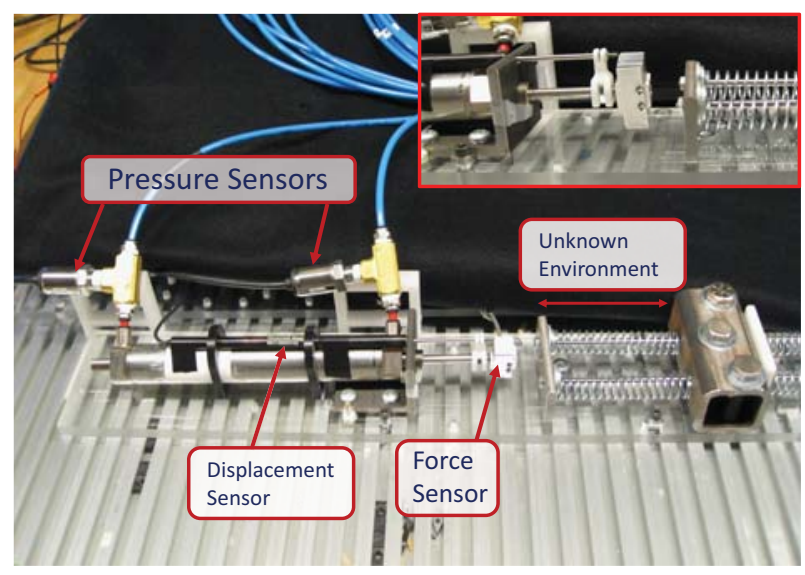

Fig. 4: The photo of the experimental setup

numerical differentiation with a first-order filter at $50 \mathrm{~Hz}$. The system was controlled by a platform of Intel i7 @ $2.80 \mathrm{GHz}$ processor with $6.00 \mathrm{~GB}$ RAM, where the control algorithm, shown in Fig. 3, were implemented using Labview. The National Instruments A/D board (NI USB-6221) was used for data acquisition.

\section{RESULTS}

The performance of the observer-based impedance controller is shown in Fig. 5 and Fig. 6. Figure 5 presents the desired and measured interaction force values, $F_{e d}$ and $F_{e}$ respectively, when the desired position of the piston, $z_{d}$, was changed to different fixed locations. The response of the proposed system was also tested under a periodically varying desired position. Fig. 6 illustrates the control performance when the position reference was varied at $0.5,1$ and $1.5 \mathrm{~Hz}$.

The contribution of the described pressure observation was analyzed by comparing the control performance of the system with the one that utilizes open-loop pressure estimation, i.e. standard system modeling. The tests with sinusoidal desired position were selected for this comparison. The control and observation accuracy are evaluated quantitatively by the averaged sum of squared errors (MSE) within one cycle in the steady-state condition as follows:

$$
\text { MSE }=\frac{1}{2} \frac{\int_{0}^{T} \boldsymbol{e}^{T} \boldsymbol{e} \mathrm{d} t}{T}
$$

where $\boldsymbol{e}$ refers to an error vector. For the evaluation of control performances the error vector has one element, the error in the interaction force. Whereas for evaluating the pressure observation accuracy, the following error vector is selected: $\boldsymbol{e}=\left[\begin{array}{ll}\left(P_{1}-\tilde{P}_{1}\right) & \left(P_{2}-\tilde{P}_{2}\right)\end{array}\right]^{T}$.

The data shown in Fig. 7 corresponds to MSE in six steady-state cycles for each estimation type, open-loop and closed-loop, and each experiment. Figure 7.a presents the mean and the variation of MSE in force. Figure 7.b shows the average MSE in pressure estimation.

Figure 8 shows the transition in the system response when the described closed-loop observer was turned on. The MSE in force for each cycle during the $0.5 \mathrm{~Hz}$ sinusoidal reference 


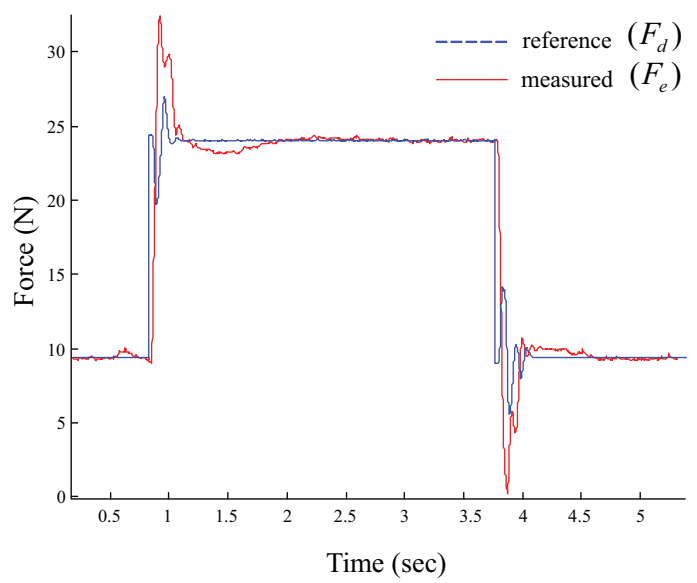

Fig. 5: The response of the system for a step change in the reference position

position test is presented in Fig. 8.a. The MSE in pressure estimation on the same test is provided in Fig. 8.b.

\section{DISCUSSION}

This work has presented the use of a new pressure observer on the control of tele-operated pneumatic systems. The observed pressures were utilized in an impedance controller to achieve an accurate force tracking on a pneumatic actuator with 7.25 meters long transmission lines.

The developed control scheme provided a interaction force tracking against an unknown environment. The system presented a time-delay issue with a rising magnitude as the reference variation frequency increases. This study was limited to $1.5 \mathrm{~Hz}$, as the contact stability was not preserved beyond. These limitations can be attributed to the significantly long transmission lines. A fixed mass transportation delay and an attenuation in the mass flow rate through the lines reduce the bandwidth of the actuation.

Figures 7 and 8 illustrate the benefit of the described observer both in pressure estimation and the control performance. The improvement in control accuracy is more significant at higher frequencies $-\% 25$ on the $1.5 \mathrm{~Hz}$ force tracking experiment- where the demand for the valve flow is greater. Pressure states are involved in the equivalent valve input; hence, they are more effective when the magnitude of the ideal flow rate is higher. On the other hand, the improvement in the pressure estimation may not follow the same trend. The described observer substantially reduced the pressure estimation errors in this study. Yet, the magnitude of the improvement depends on the quality of the valve calibration within the given interval of valve inputs for a specific operation. The advantage of utilizing the observer can also be viewed by the rate of error convergences, shown in Fig. 8. A rapid improvement in the pressure estimation initiates a downward slope in the force error when the observation is turned on.

\section{CONCLUSION}

This study presented a pressure observer based impedance controller for pneumatic systems with long transmission

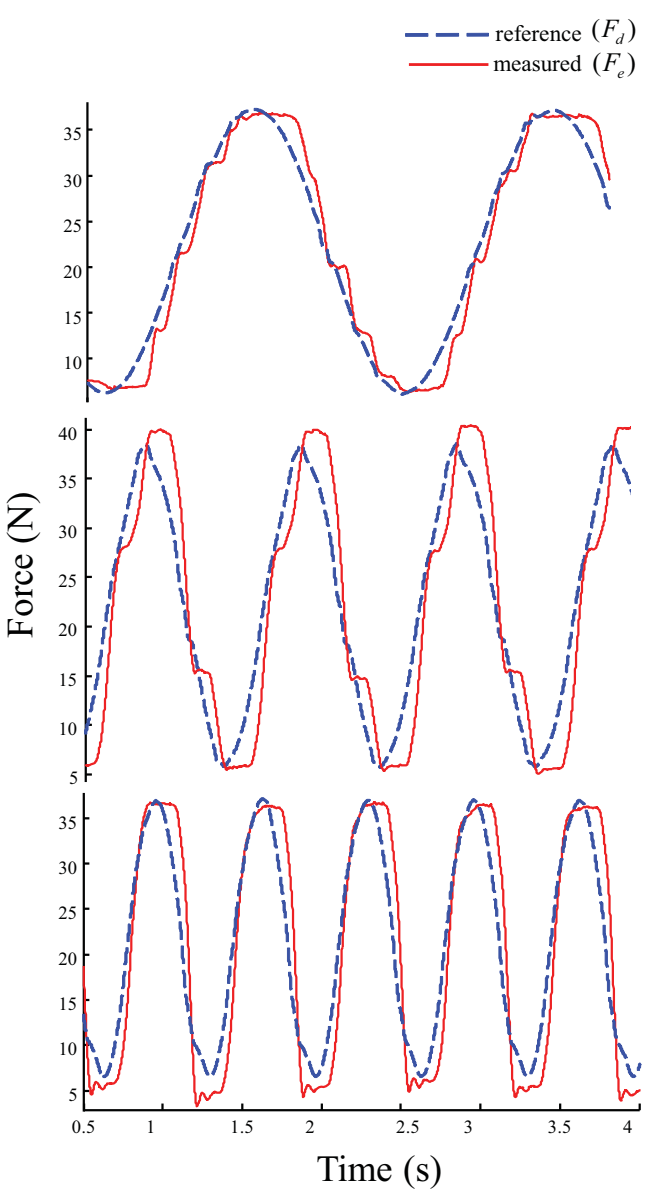

Fig. 6: The response of the system for sinusoidal reference variation. $0.5 \mathrm{~Hz}-1 \mathrm{~Hz}-1.5 \mathrm{~Hz}$ reference signal from top to bottom

lines. The adverse effects of the transmission lines in pressure estimation and the lack of direct pressure feedback from the actuator are compensated by the described observer. The experiments on a system with 7.25 meters long transmission lines indicated that the use of the observer improves the accuracy of the force tracking as well. This study can be extended by analyzing the range of impedance characteristics that can be provided by the tested system. The motivation for the developed algorithm comes from the potential of pneumatically driven systems for robotic rehabilitation in MRI. The described algorithm will be applied on an MRIcompatible haptic interface with long transmission lines in the future.

\section{REFERENCES}

[1] K. Kawahira, T. Noma, J. Iiyama, S. Etoh, A. Ogata, and M. Shimodozono, "Improvements in limb kinetic apraxia by repetition of a newly designed facilitation exercise in a patient with corticobasal degeneration," International Journal of Rehabilitation Research, vol. 32, pp. 178-83, 2009.

[2] H. Krebs, L. Dipietro, S. Levy-Tzedek, S. E. Fasoli, A. RykmanBerland, J. Zipse, J. Fawcett, J. Stein, H. Poizner, A. C. Lo, et al., "A paradigm shift for rehabilitation robotics," Engineering in Medicine and Biology Magazine, IEEE, vol. 27, no. 4, pp. 61-70, 2008. 


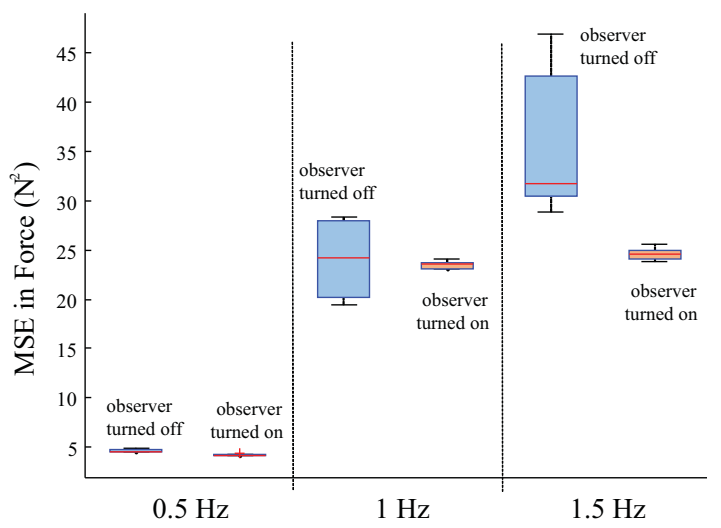

(a)

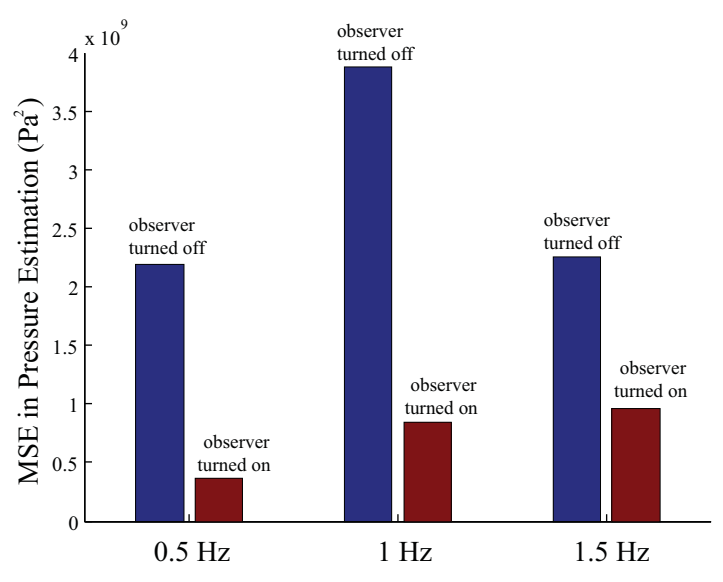

(b)

Fig. 7: Mean squared error (MSE) for the sinusoidal reference position tests (a)in output force (b) in pressure estimation

[3] J. Ueda, L. Lacey, M. Turkseven, M. Shinohara, A. Maliki, and I. Kovalenko, "Robotic rehabilitation exercise in hemiparetic limbs based on functional synchronization of voluntary and involuntary nerve impulses," in 2014 IEEE International Workshop on Advanced Robotics and its Social Impacts, 2014.

[4] R. Gassert, R. Moser, E. Burdet, and H. Bleuler, "Mri/fmri-compatible robotic system with force feedback for interaction with human motion," Mechatronics, IEEE/ASME Transactions on, vol. 11, no. 2, pp. 216-224, 2006.

[5] J. F. Schenck, "'the role of magnetic susceptibility in magnetic resonance imaging: Mri magnetic compatibility of the first and second kinds", medical physics," Vol., vol. 23, pp. 815-997, 1996.

[6] R. Gassert, A. Yamamoto, D. Chapuis, L. Dovat, H. Bleuler, and E. Burdet, "Actuation methods for applications in mr environments," CONCEPTS IN MAGNETIC RESONANCE PART B MAGNETIC RESONANCE ENGINEERING, vol. 29, no. 4, p. 191, 2006.

[7] N. Yu, C. Hollnagel, A. Blickenstorfer, S. S. Kollias, and R. Riener, "Comparison of mri-compatible mechatronic systems with hydrodynamic and pneumatic actuation," Mechatronics, IEEE/ASME Transactions on, vol. 13, no. 3, pp. 268-277, 2008.

[8] M. Turkseven and J. Ueda, "Design of an mri compatible haptic interface," in Intelligent Robots and Systems (IROS), 2011 IEEE/RSJ International Conference on, Sept 2011, pp. 2139-2144.

[9] N. Yu, W. Murr, A. Blickenstorfer, S. Kollias, and R. Riener, "An fmri compatible haptic interface with pneumatic actuation," in Rehabilitation Robotics, 2007. ICORR 2007. IEEE 10th International Conference on. IEEE, 2007, pp. 714-720.

[10] N. Gulati and E. Barth, "A Globally Stable, Load-Independent Pressure Observer for the Servo Control of Pneumatic Actuators," IEEE/ASME Transactions on Mechatronics, vol. 14, no. 3, pp. 295-

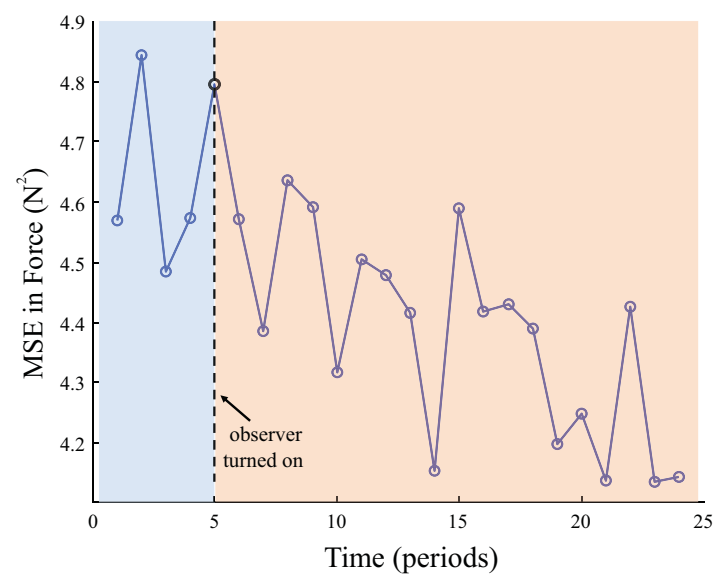

(a)

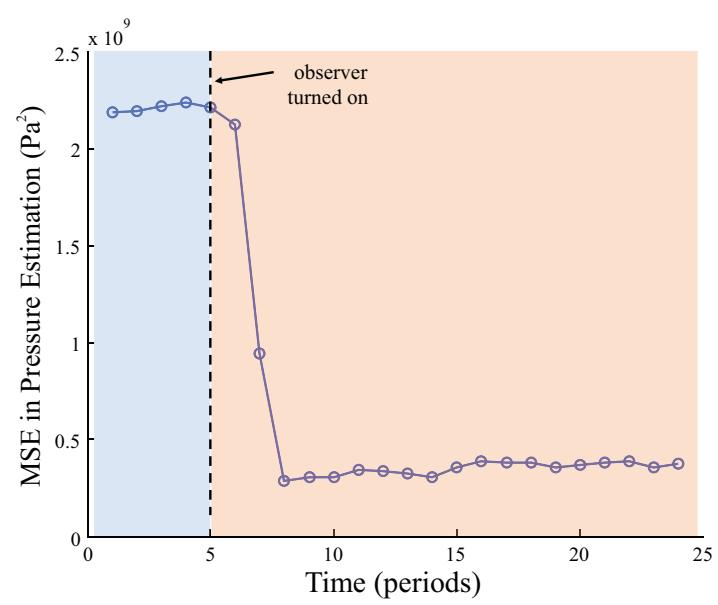

(b)

Fig. 8: Mean squared error (MSE) for $0.5 \mathrm{~Hz}$ sinusoidal reference position (a)in output force (b) in pressure estimation

306, June 2009.

[11] E. E. ELMADBOULY t and M. ABDULSADEK NOURZ, "MODELLING, SIMULATION AND SENSITIVITY ANALYSIS OF A STRAIGHT PNEUMATIC PIPELINE," Energy Convers. Mgmt, vol. 35, no. I, pp. 61-77, 1994.

[12] S. V. Krichel and O. Sawodny, "Non-linear friction modelling and simulation of long pneumatic transmission lines," Mathematical and Computer Modelling of Dynamical Systems, vol. 20, no. 1, pp. 23-44, Jan. 2014. [Online]. Available: http://www.tandfonline.com/doi/abs/10.1080/13873954.2013.811268

[13] M. Turkseven and J. Ueda, "An asymptotically stable pressure observer based on load and displacement sensing for pneumatic actuators with long transmission lines," IEEE Transactions on Mechatronics, submitted in November, 2014.

[14] P. Beater, "6 Modelling of Long Lines," no. Hennig 1982, pp. 473477, 2004.

[15] ISO6358:1989, "Pneumatic fluid power - components using compressible fluids - determination of flow-rate characteristics," 1989.

[16] E. Richer and Y. Hurmuzlu, "A High Performance Pneumatic Force Actuator System : Part I ? Nonlinear Mathematical," vol. 122, no. September, 2000.

[17] N. Hogan, "Impedance control: An approach to manipulation: Part iiimplementation," Journal of dynamic systems, measurement, and control, vol. 107, no. 1, pp. 8-16, 1985.

[18] Q. Ha, Q. Nguyen, D. Rye, and H. Durrant-Whyte, "Impedance control of a hydraulically actuated robotic excavator," Automation in construction, vol. 9, no. 5, pp. 421-435, 2000.

[19] J.-J. E. Slotine, W. Li, et al., Applied nonlinear control. Prentice-hall Englewood Cliffs, NJ, 1991, vol. 199, no. 1. 